\title{
Neuroblastoma in Late Adolescence: Case Report and Review of Literature
}

\author{
${ }^{1}$ Shreyamsa Manjunath, ${ }^{2}$ Sasi Mouli, ${ }^{3}$ Kul R Singh, ${ }^{4}$ Chanchal Rana, ${ }^{5}$ Pooja Ramakant, ${ }^{6}$ Anand Mishra
}

\begin{abstract}
Neuroblastoma is an embryonal cancer of the peripheral sympathetic nervous system. It is the most common extracranial tumor of childhood and the third most common tumor overall. Neuroblastoma arises from cells of primordial neural crest. The natural history of this disease is very heterogeneous and extends from spontaneous regression to aggressive metastatic disease. Neuroblastoma in older children is extremely rare and it carries a bad prognosis. We present a case of a 15-year-old boy with unresectable neuroblastoma who was subjected to neoadjuvant chemotherapy which rendered the tumor resectable. The case highlights importance of neoadjuvant therapy in neuroblastomas, need for multimodality involvement and therapeutic challenges faced by phycisians in successfully treating aggressive neuroblastomas.
\end{abstract}

Keywords: Adrenal tumor, Late adolescence, Neuroblastoma, Sympathetic nervous system tumors.

How to cite this article: Manjunath S, Mouli S, Singh KR, Rana C, Ramakant P, Mishra A. Neuroblastoma in Late Adolescence: Case Report and Review of Literature. World J Endoc Surg 2018;10(3):157-162.

\section{Source of support: Nil}

Conflict of interest: None

\section{INTRODUCTION}

Neuroblastomas are embryonal cancers of the peripheral sympathetic nervous system. They arise from cells of primordial neural crest which are the precursors of sympathetic ganglia and adrenal medulla. Neuroblastoma is the most common extracranial tumor of childhood and the third most common tumor overall. ${ }^{1}$ Its natural history extends from spontaneous regression to relentless progression. Neuroblastoma is an ideal archetype of a disease where clinico-biological data are effectively utilized for customizing therapy for different patient groups. Overall survival is generally good for patients with low and intermediate risk tumors while in those with high-risk tumors, the survival is less than $40 \%{ }^{2}$

\footnotetext{
${ }^{1,2}$ Senior Resident, ${ }^{3,4}$ Assistant Professor, ${ }^{5}$ Associate Professor, ${ }^{6}$ Professor

1-3,5,6 Department of Endocrine and Breast Surgery, King Georges Medical University, Lucknow, Uttar Pradesh, India

${ }^{4}$ Department of Pathology, King Georges Medical University, Lucknow, Uttar Pradesh, India

Corresponding Author: Shreyamsa Manjunath, Senior Resident, Department of Endocrine and Breast Surgery, King Georges Medical University, Lucknow, Uttar Pradesh, India, e-mail: shreyamsam@gmail.com
}

Neuroblastoma presenting after the age of fifteen years is remarkably rare and it poses a great therapeutic challenge. We discuss the case of neuroblastoma in late adolescence and its management along with a review of the literature.

\section{CASE REPORT}

A 15-year-old boy presented with complaints of intermittent, vague abdominal pain for one year and gradual distention of abdomen for ten months. He also had fatigue, significant weight loss and generalized malaise for five months. Family history was not contributory. General examination revealed pallor. A non-tender, firm mass occupying all quadrants was evident on palpation of the abdomen. There was no free fluid. The biochemical assessment revealed hemoglobin of 6.9 gram \%, serum LDH of $2539 \mathrm{U} / \mathrm{L}$, normal serum cortisol and overnight dexamethasone suppression test (ONDST) levels, urinary metanephrines of $137.6 \mu \mathrm{g} \%$ and urinary normetanephrine of $6295.9 \mu \mathrm{g} \%$. A contrast-enhanced computed tomography (CECT) of the abdomen showed a mass measuring $24.1 \times 19.8 \times 18.8 \mathrm{~cm}$. It was displacing the liver anterosuperiorly with a distorted surface (Fig. 1). The mass also compressed the spleen with loss of fat planes. The head of the pancreas, uncinate process of the pancreas and the common bile duct were also compressed by the tumor. There was significant compression of the aorta, IVC, right renal vein and portal vein with no tumor thrombus (Fig. 2). Multiple enlarged retroperitoneal nodes were also seen.

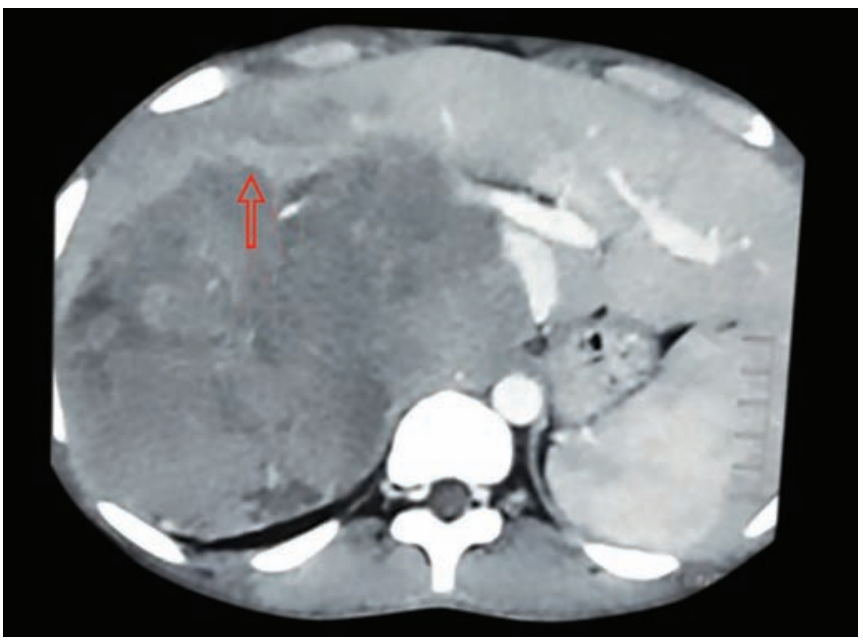

Fig. 1: Tumor abutting the liver 


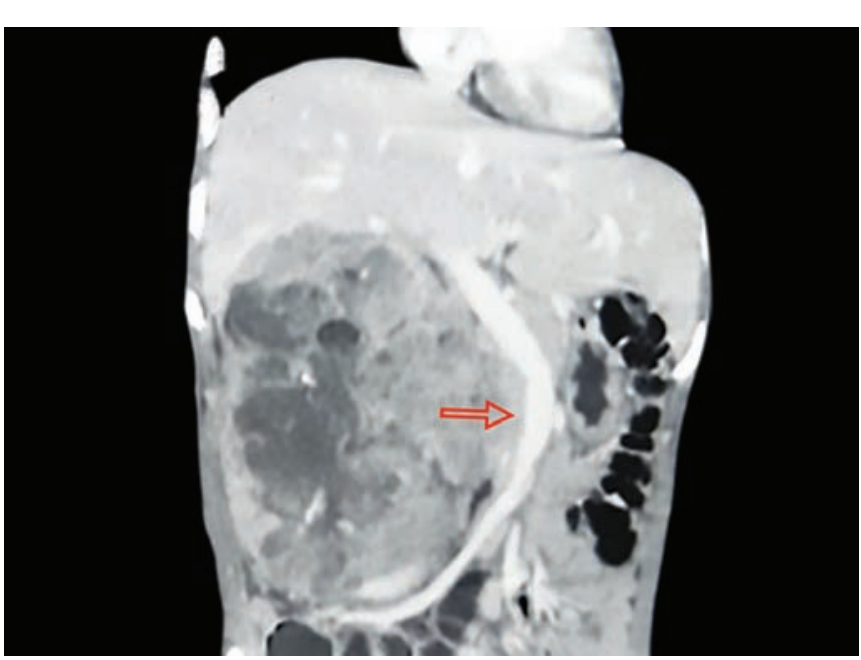

Fig. 2: Tumor displacing IVC

Core needle biopsy showed predominant areas of necrosis with clusters of atypical cells. Homer-Wright pseudorosettes were prominently recognizable (Fig. 3). IHC analysis showed strongly positive synaptophysin and moderately positive NSE (Fig. 4). LCA, WT1, and CD99 were negative, ruling out lymphomas, Wilm's tumor, and GIST. Metaiodobenzylguanidine (MIBG) scan with Single-photon emission computed tomography (SPECT) showed heterogenous tracer concentration in the right hypochondrium with multiple hypodense lesions scattered throughout liver parenchyma, suggestive of tumor arising from the right adrenal with possible liver metastasis. Bone marrow aspiration study was negative for marrow infiltration. The patient was started on neoadjuvant chemotherapy commensurate with SIOPEN protocol after a multi-disciplinary tumor board discussion. ${ }^{3}$ Five cycles of cisplatin, etoposide, doxorubicin and cyclophosphamide regimen was administered. Posttherapy scans showed more than $50 \%$ reduction in size (Fig. 5).

The patient was optimized and alpha blockade started with prazosin. The patient underwent right open adrenalectomy, where a large mass was found adherent to the inferior surface of the liver, right kidney, and the omentum. Upper pole of the kidney was encased but not infiltrated, with enlarged renal hilar nodes. Medially the mass was adherent to the inferior vena cava. Few nodules (largest measuring $3 \times 3 \mathrm{~cm}$ ) were found in the omentum, which was excised (Figs 6 and 7). The patient had uneventful intra- and post-operative periods. He was discharged on postoperative day 7. Postoperative histopathology report showed right adrenal gland with an intact capsule and focal residual tumor (neuroblastoma) and noticeable chemotherapy-induced changes. The omental nodule was positive for focal tumor deposits. He received external beam radiation to the tumor bed. The

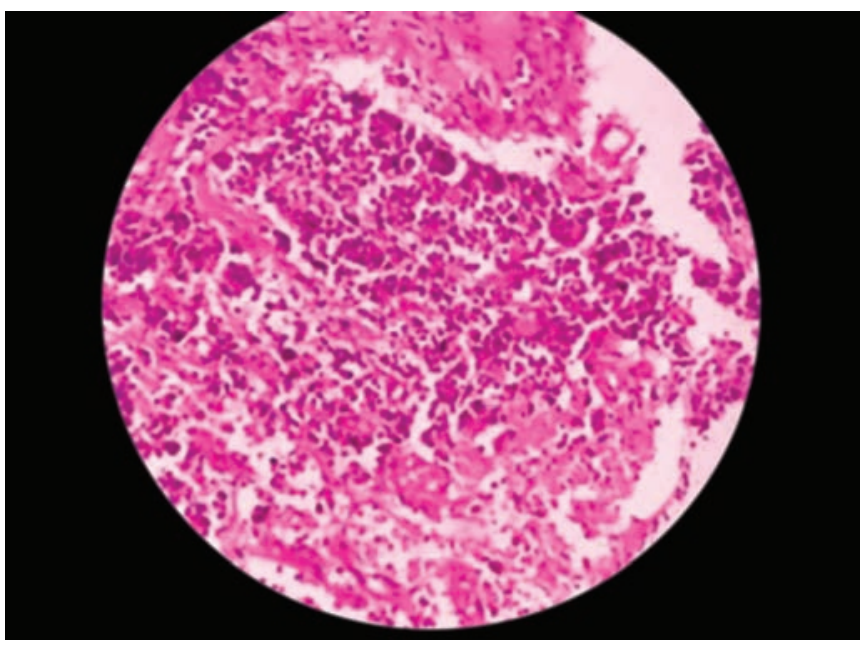

Fig. 3: Histopathology

patient continues to remain asymptomatic in 6 months of follow-up.

\section{REVIEW OF LITERATURE}

Neuroblastoma is the most frequent extracranial tumor of childhood and the most frequently diagnosed malignancy in infancy. ${ }^{1,2}$ It is a tumor which originates from embryonal remnants of the sympathoadrenal lineage of neural crest cells, hence can develop in any part of the sympathetic nervous system. ${ }^{1,4}$ Along with ganglioneuroma and ganglioneuroblastoma, they constitute the peripheral neuroblastic tumor family. ${ }^{2}$ They make up 7 to $10 \%$ of all childhood malignancies and account for $15 \%$ of all pediatric malignancy-related deaths. ${ }^{1,3}$ More than $75 \%$ of the tumors are diagnosed before 4 years of age (median-22 months) and less than 5\% of all neuroblastomas are seen after the age of 10 years. ${ }^{5}$ It is exceptionally rare $(0.2 \%)$ in the age group of 15 to 18 years. ${ }^{6}$ The incidence in Asia follows the worldwide data, while that in India is $3.2 \%{ }^{6}$

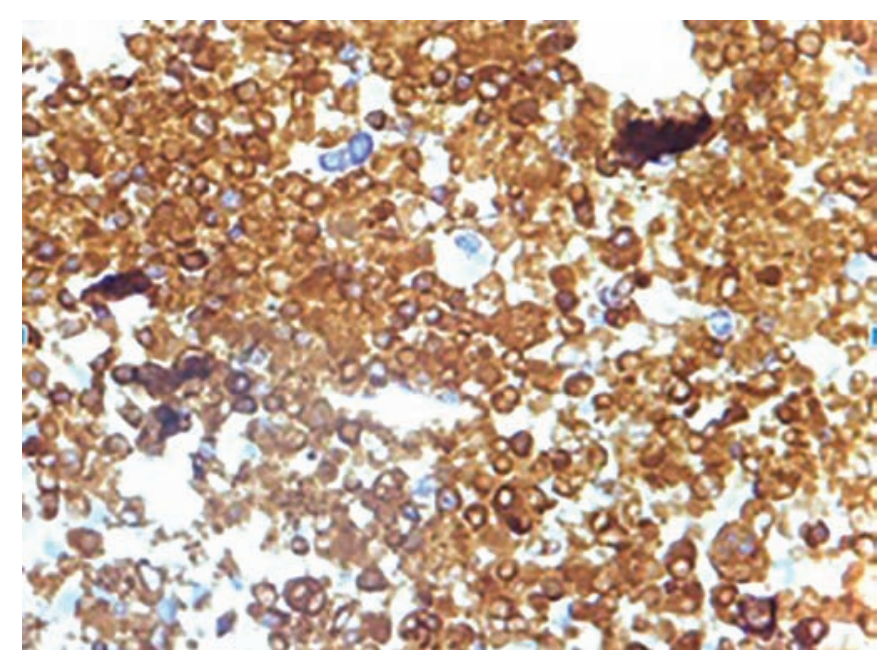

Fig. 4: IHC showing synaptophysin positivity 

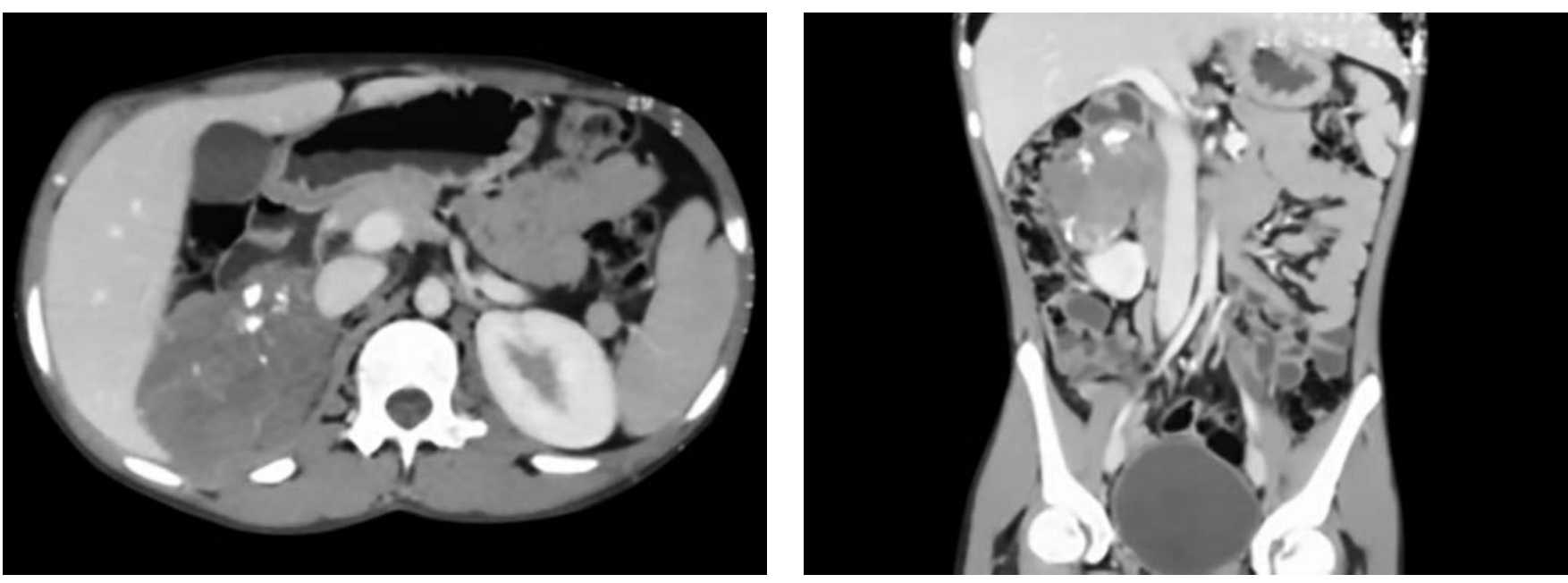

Figs 5A and B: (A) Response to neoadjuvant chemotherapy; (B) Response to neoadjuvant chemotherapy

Many studies have shown a possible relation between prenatal exposure of the mother and the risk of neuroblastoma in offspring. Maternal consumption of alcohol/ tobacco, diuretics, oral contraceptive pills, codeine and preconception exposure of the mother to wood dust, diesel fuels, lacquer thinner, and turpentine are shown to increase the risk. ${ }^{2}$ Neuroblastomas are associated with many genetic/ chromosomal aberrations, which serve as molecular markers for risk assignment as well as prognosis. MYCN oncogene amplification on chromosome 2 is seen in about $25 \%$ of cases and is associated with aggressive tumor progression and a poor prognosis. ${ }^{7}$ The most common chromosomal abnormality found is the deletion of short arm of chromosome 1 and it is associated with poor prognosis. Neurotrophic receptor gene products Trk A and Trk $\mathrm{C}$ portend good prognosis while Trk B indicates an adverse prognosis. Increased telomerase expression, lack of CD44 expression and increased serum LDH are also associated with poor prognosis. Familial neuroblastomas are seen in less than $10 \%$ of the cases. ${ }^{8}$ They usually occur in neurofibromatosis type I, Li Fraumeni syndrome, Beckwith-Wiedemann syndrome,

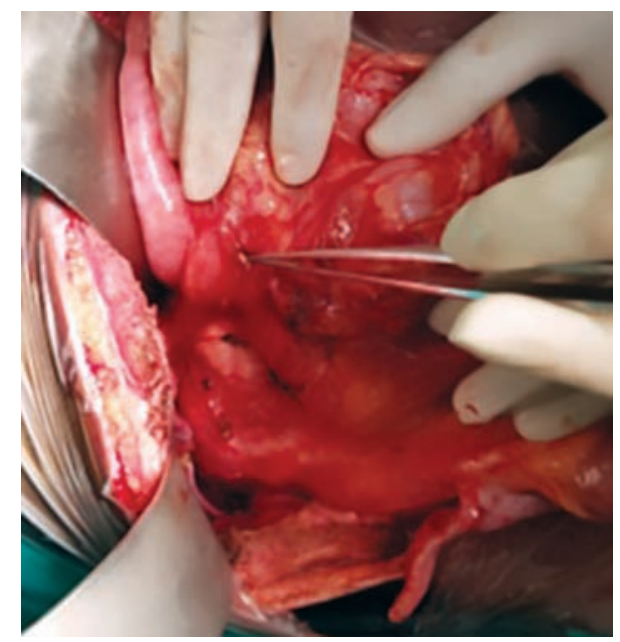

Fig. 6: Omental deposits
Noonan's syndrome and exhibit mutations in $A L K$ and PHOX2B genes. ${ }^{8,9}$

Pathologically, neuroblastomas are a part of the "small blue round cell tumors" of childhood. A typical neuroblastoma consists of small, uniform cells with scant cytoplasm and dense hyperchromatic nuclei. HomerWright pseudorosettes are seen in more than half of the cases and are made up of neuroblastic cells surrounding areas of eosinophilic neuritic processes called neuropil. ${ }^{2}$ Immunohistochemical diagnosis of neuroblastoma is established by demonstrating positive staining for neural markers like neuron-specific enolase, synaptophysin, neurofilament protein, ganglioside GD2, chromogranin A, and tyrosine hydroxylase. ${ }^{2}$ These also help in differentiating neuroblastomas from other small blue round cell tumors.

More than half of the neuroblastomas occur in the abdomen (Table 1). ${ }^{8}$ Clinical manifestation of neuroblastoma reflects the tumor site, extent of disease and presence of paraneoplastic syndromes.

Nearly fifty percent of all patients are diagnosed with metastases at presentation. Patients with localized disease

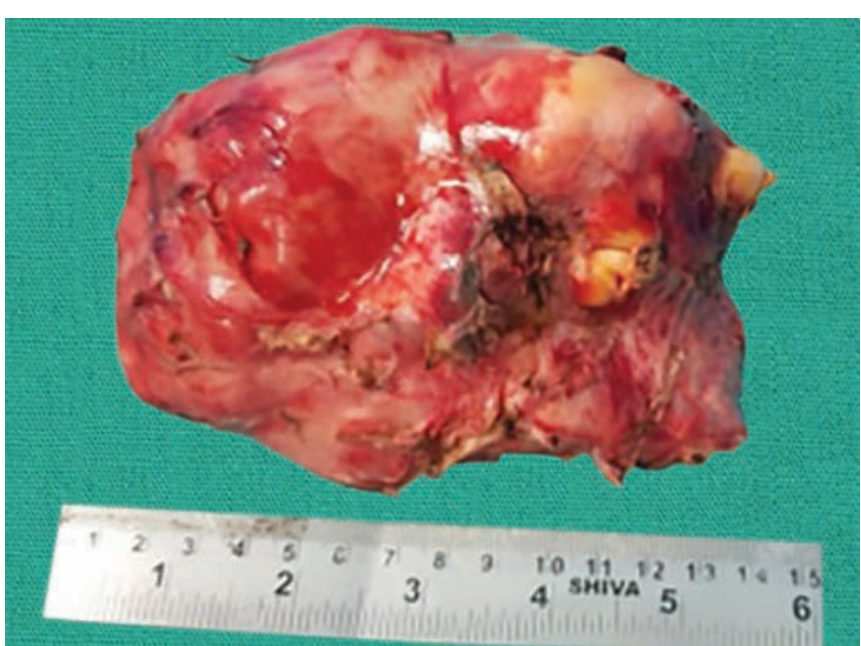

Fig. 7: Resected specimen 


\begin{tabular}{ll}
\hline & Table 1: Distribution of neuroblastoma \\
\hline Site & Incidence \\
\hline Adrenals & $40 \%$ \\
Paraspinal region & $25 \%$ \\
Thorax & $15 \%$ \\
Pelvis & $5 \%$ \\
Cervical & $3 \%$ \\
\hline
\end{tabular}

are seldom symptomatic, with the diagnosis made incidentally while under evaluation for unrelated conditions. Disease dissemination occurs via both hematogenous and lymphatic routes. Bone marrow, bones, and liver are usual sites of hematogenous spread, with an increased predilection for the skull, orbital and metaphyseal bone sites. ${ }^{2,4}$ The clinical manifestations of neuroblastoma can be broadly categorized as direct mass effects by the tumor, humoral effects by excess catecholamines and nonspecific manifestations due to metastases. Neuroblastomas are associated with numerous paraneoplastic syndromes like Pepper syndrome, opsoclonus-myoclonus, Hutchinson's syndrome, and Kerner Morrison syndrome. ${ }^{10-12}$

The International Neuroblastoma Staging System (INSS) recommends the following investigations for the appropriate diagnosis of neuroblastoma: $:^{13}$

- Imaging (CT or MRI) of the primary tumor region, to assess the extent of the primary disease

- Histological evaluation of a primary tumor or metastases

- Bone marrow aspirate (preferably at two different sites), to rule out/ establish bone marrow infiltration.

- Meta-iodo-benzyl-guanidine (I-MIBG) scintigraphy, to assess primary tumor site and to detect foci of metastases. I-MIBG, the guanethidine derivative analog of norepinephrine is a unique molecule, as it is specifically taken up and stored in tumors of sympathetic nervous system origin. Due to its high specificity and sensitivity in neuroblastoma, MIBG scintigraphy is a recommended investigation for staging and response evaluation. ${ }^{14}$

- Urinary catecholamine metabolite level assays

Additional investigations like serum ferritin level, serum LDH and neuron-specific enolase (NSE) aid in establishing the diagnosis

The International Neuroblastoma Staging System (INSS) was devised to stage patients with neuroblastoma, based on the surgeon's judgment of resectability (Table 2). ${ }^{13}$

The International Neuroblastoma Risk Group (INRG) classification system was developed in 2009 by defining homogeneous pre-treatment patient cohorts. ${ }^{8,14,15}$ This classification defines risk groups more homogeneously. It also incorporates radiological criteria on diagnosis and assigns risk factors for surgery based on the radiological findings.
Table 2: International Neuroblastoma Staging System

Stage 1 Localized tumor, complete gross excision with or without microscopic residual disease; representative ipsilateral lymph nodes negative for tumor microscopically

Stage 2A Localized tumor, incomplete gross resection; representative ipsilateral nonadherent lymph nodes negative for tumor microscopically

Stage 2B Localized tumor with or without complete gross excision with ipsilateral nonadherent lymph nodes positive for tumor; contralateral lymph nodes must be negative microscopically

Stage 3 Unresectable unilateral tumor infiltrating across the midline with or without regional lymph node involvement, localized unilateral tumor with contralateral regional lymph node involvement, or midline tumor with bilateral extension by infiltration (unresectable) or by lymph node involvement

Stage 4 Any primary tumor with dissemination to distant lymph nodes, bone marrow, bone, skin, liver, or other organs (except stage 4S)

Stage 4S Localized primary tumor (as defined for stage 1, $2 \mathrm{~A}$, or $2 \mathrm{~B}$ ) with dissemination limited to skin, liver, or bone marrow (limited to infants less than one year of age)

Most important prognostic factors include age at presentation, stage and MYCN amplification. Two distinct patterns of disease are defined by these factors. The first one is neuroblastoma in infancy and early childhood: some patients experience spontaneous regression of the disease or have excellent survival with minimal treatment, provided there is no MYCN amplification. ${ }^{16,17}$ In contrast, children who have metastatic tumors, harbor MYCN amplification or aged more than 18 months at diagnosis have an unfavorable outcome. ${ }^{18,19}$ Between these two extremes, there is an "intermediate group" with less defined features. Other pivotal markers like histopathology (based on Shimada classification), tumor ploidy, and chromosomal anomalies including a gain of chromosomes 1q, 17 can help in better definition of prognosis and consequently enable customization of treatment strategies to individuals in this intermediate group. ${ }^{7}$ Neuroblastoma in adolescents and adults is very rare, and in these patients have a dismal outcome. ${ }^{5,19}$

Most patients undergo multimodality treatment with surgery, chemotherapy, radiotherapy and/or biotherapy. Rarely, observation-only is apt in select few. These modalities are used alone or in combination based on prognostic factors present and subsequent risk-group stratification. Surgery plays an important role both in diagnosis and treatment. ${ }^{20}$ Surgery may be the only treatment required if complete excision can be successfully achieved. In unresectable tumors, tissue for histological confirmation can be obtained. Since neuroblastoma has a high affinity for the lymphatic system, surgical exploration of loco-regional abdominal and pelvic nodal basins should be performed. Chemotherapy is the cornerstone 


\begin{tabular}{|c|c|c|c|}
\hline & Low risk & $\begin{array}{l}\text { Intermediate } \\
\text { risk }\end{array}$ & High risk \\
\hline Prognosis & Excellent & $\begin{array}{l}\text { Excellent for } \\
\text { favorable } \\
\text { histology }\end{array}$ & Poor \\
\hline Treatment & $\begin{array}{l}\text { Curative } \\
\text { surgery }\end{array}$ & Multimodality & Multimodality \\
\hline $\begin{array}{l}\text { Adjuvant } \\
\text { Therapy }\end{array}$ & $\begin{array}{l}\text { Not indicated } \\
\text { even in R1 } \\
\text { resection } \\
\text { (unless } \\
\text { associated } \\
\text { with } \\
\text { unfavorable } \\
\text { histology) }\end{array}$ & $\begin{array}{l}\text { Chemotherapy } \\
\text { with/ without } \\
\text { radiotherapy }\end{array}$ & $\begin{array}{l}\text { Induction } \\
\text { (chemotherapy)- } \\
\text { Local control } \\
\text { (Surgery)- } \\
\text { Consolidation } \\
\text { (chemotherapy) } \\
\text { and Residual } \\
\text { disease control } \\
\text { (Radiation) }\end{array}$ \\
\hline $\begin{array}{l}\text { Survival in } \\
\text { unfavorable } \\
\text { histology }\end{array}$ & Good & $\begin{array}{l}\text { Reduced } \\
\text { overall } \\
\text { survival }\end{array}$ & $\begin{array}{l}\text { Least overall } \\
\text { survival }\end{array}$ \\
\hline $\begin{array}{l}\text { Treatment } \\
\text { of } \\
\text { recurrence }\end{array}$ & $\begin{array}{l}\text { Salvage } \\
\text { chemotherapy }\end{array}$ & $\begin{array}{l}\text { Salvage } \\
\text { chemotherapy }\end{array}$ & $\begin{array}{l}\text { Consolidation } \\
\text { with high dose } \\
\text { chemotherapy } \\
\text { and stem cell } \\
\text { rescue }\end{array}$ \\
\hline Radiation & Not indicated & Indicated & Indicated \\
\hline
\end{tabular}

of treatment for locally advanced and metastatic disease and is also indicated in low-risk patients with involvement of vital organs. In the current SIOPEN protocols, irradiation of the primary tumor bed is recommended after surgery in patients with MYCN amplified tumors, stage 4 disease and stage 3 tumors with unfavorable biology and/or histology. Radio-metabolic therapy with I-131 tagged to benzylguanidine (I-131 MIBG) is also used with variable results. ${ }^{21}$ Radionuclide targeted therapy with peptides against somatostatin receptors tagged to yttrium-90 (Y-90) and lutetium-177 (Lu-177) are under investigation. ${ }^{22}$ Summary of treatment and outcomes of neuroblastomas are outlined in Table $3 .{ }^{8}$ Stage $4 \mathrm{~S}$ neuroblastoma undergoes spontaneous regression in most cases when not associated with MYCN amplification.

Our patient had many positive risk factors, namely age (15 years), higher stage (INSS stage 3 ) which places him in the high-risk category. Chromosomal studies were not performed due to logistic limitations and patient's socio-economic status. An excellent response to neoadjuvant chemotherapy was followed by complete surgical extirpation and irradiation of the tumor bed, as per standard protocols.

In conclusion, neuroblastoma rarely occurs in adolescents and is extremely rare in older children. These tumors are of the advanced stage with an unfavorable prognosis and carry a high risk of metastasis. Meticulously planned multimodality intervention is the key to successful treatment and good outcomes in these patients. An advance in molecular studies with the goal of patient-oriented therapies and to identify critical targets for treatment is the need of the hour. It is imperative that the extensive knowledge of neuroblastoma biology is used for developing novel therapies for high-risk neuroblastoma.

\section{REFERENCES}

1. Maris JM, Hogarty MD, Cohn SL. "Neuroblastoma." The Lancet, 2007;369(9579):2106-2120.

2. Park JR, Eggert A, Caron H. Neuroblastoma: biology, prognosis, and treatment. Hematology/oncology clinics of North America. 2010 Feb 1;24(1):65-86.

3. Parikh NS, Howard SC, Chantada G. SIOP-PODC adapted risk stratification and treatment guidelines: Recommendations for neuroblastoma in low and middle income settings. Pediatr Blood Cancer, 2015;62(8):1305-1316.

4. Zage PE, Ater JL Neuroblastoma. In: Kliegman, Stanton, St Geme, Schor, editors. Nelson textbook of paediatrics. 20th edition; New York: Elsevier: 2016. Chapter 498, 2461-2464.

5. Mossé YP, Deyell RJ, Berthold F, Nagakawara A, Ambros $\mathrm{PF}$, Monclair T, et al. Neuroblastoma in older children, adolescents and young adults: a report from the International Neuroblastoma Risk Group project. Pediatric blood \& cancer. 2014 Apr;61(4):627-635.

6. Steliarova-Foucher E, Colombet M, Ries LA, Moreno F, Dolya A, Bray F, et al. International incidence of childhood cancer, 2001-10: a population-based registry study. The Lancet Oncology. 2017 Jun 1;18(6):719-731.

7. Vo KT, Matthay KK, Neuhaus J, London WB, Hero B, Ambros $\mathrm{PF}$, et al. Clinical, biologic, and prognostic differences on the basis of primary tumor site in neuroblastoma: a report from the international neuroblastoma risk group project. Journal of clinical oncology. 2014 Oct 1;32(28):3169-3176.

8. Cohn SL, Pearson AD, London WB, Monclair T, Ambros PF, Brodeur GM, et al. The International Neuroblastoma Risk Group (INRG) classification system: an INRG task force report. Journal of clinical oncology. 2009 Jan 10;27(2):289-297.

9. Irwin MS, Park JR. Neuroblastoma: paradigm for precision medicine. Pediatric Clinics of North America. 2015 Feb;62(1):225-256.

10. Castleberry RP. Biology and treatment of neuroblastoma. Pediatric Clinics of North America. 1997 Aug;44(4):919-937.

11. Krug P, Schleiermacher G, Michon J, Valteau-Couanet D, Brisse $\mathrm{H}$, Peuchmaur $\mathrm{M}$, et al. Opsoclonus-myoclonus in children associated or not with neuroblastoma. european journal of paediatric neurology. 2010 Sep 1;14(5):400-409.

12. Han W, Wang HM. Refractory diarrhea: A paraneoplastic syndrome of neuroblastoma. World Journal of Gastroenterology: WJG. 2015 Jul 7;21(25):7929-7932.

13. Brodeur GM, Pritchard J, Berthold F, Carlsen NL, Castel V, Castelberry RP, et al. Revisions of the international criteria for neuroblastoma diagnosis, staging, and response to treatment. J Clin Oncol. 1993 Aug;11(8):1466-1477.

14. Brisse HJ, McCarville MB, Granata C, Krug KB, WoottonGorges SL, Kanegawa K, et al. Guidelines for imaging and staging of neuroblastic tumors: consensus report from the International Neuroblastoma Risk Group Project. Radiology. 2011 Oct;261(1):243-257.

15. Monclair T, Brodeur GM, Ambros PF, Brisse HJ, Cecchetto G, Holmes K, et al. The International Neuroblastoma Risk 
Group (INRG) staging system: an INRG Task Force report. J Clin Oncol. 2009 Jan 10;27(2):298-303.

16. Cheung NK, Dyer MA. Neuroblastoma: developmental biology, cancer genomics and immunotherapy. Nature Reviews Cancer. 2013 Jun;13(6):397-411.

17. Pinto NR, Applebaum MA, Volchenboum SL, Matthay KK, London WB, Ambros PF, et al. Advances in risk classification and treatment strategies for neuroblastoma. J. Clin. Oncol. 2015; 33: pp. 3008-3017

18. Castel V, Villamón E, Cañete A, Navarro S, Ruiz A, Melero $\mathrm{C}$, et al. Neuroblastoma in adolescents: genetic and clinical characterisation. Clinical and Translational Oncology. 2010 Jan 1;12(1):49-54.

19. Agarwala S, Mandelia A, Bakhshi S, Srinivas M, Bajpai M, Gupta AK, et al. Neuroblastoma: Outcome over a 14 year period from a tertiary care referral centre in India. Journal of pediatric surgery. 2014 Aug 1;49(8):1280-1285

20. Kubota, M. The role of surgery in the treatment of neuroblastoma. Surg Today. 2010;40(6):526-532.

21. Yanik GA, Villablanca JG, Maris JM, Weiss B, Groshen S, Marachelian A, et al. 131I-metaiodobenzylguanidine with intensive chemotherapy and autologous stem cell transplantation for high-risk neuroblastoma. A new approaches to neuroblastoma therapy (NANT) phase II study. Biology of Blood and Marrow Transplantation. 2015 Apr 1;21(4):673-681.

22. Kong G, Hofman MS, Murray WK, Wilson S, Wood P, Downie $\mathrm{P}$, et al. Initial experience with gallium-68 DOTA-octreotate PET/CT and peptide receptor radionuclide therapy for pediatric patients with refractory metastatic neuroblastoma. Journal of pediatric hematology/oncology. 2016 Mar 1;38(2):87-96. 